\title{
Imaging body composition in cancer patients: visceral obesity, sarcopenia and sarcopenic obesity may impact on clinical outcome
}

\author{
Connie Yip ${ }^{1,2} \cdot$ Charlotte Dinkel $^{1}$ - Abhishek Mahajan ${ }^{1} \cdot$ Musib Siddique $^{1}$. $^{1}$ \\ Gary J. R. Cook ${ }^{1,3} \cdot$ Vicky Goh ${ }^{1,4,5}$
}

Received: 23 April 2015 /Revised: 27 May 2015 / Accepted: 29 May 2015 / Published online: 13 June 2015

(C) The Author(s) 2015. This article is published with open access at Springerlink.com

\begin{abstract}
In recent years, there has been increasing interest in the influence of body composition on oncological patient outcomes. Visceral obesity, sarcopenia and sarcopenic obesity have been identified as adverse factors in cancer patients. Imaging quantification of body composition such as lean muscle mass and fat distribution is a potentially valuable tool. This review describes the following imaging techniques that may be used to assess body composition: dual-energy X-ray absorptiometry (DXA), computed tomography (CT) and magnetic resonance imaging (MRI). CT and MRI are acquired as part of oncological patient care, thus providing an opportunity to integrate body composition assessment into the standard clinical pathway and allowing supportive care to be commenced as appropriate to improve outcome.
\end{abstract}

Vicky Goh

vicky.goh@kcl.ac.uk

1 Division of Imaging Sciences \& Biomedical Engineering, King's College London, London, UK

2 Department of Radiation Oncology, National Cancer Centre, Singapore, Singapore

3 PET Imaging Centre, Guy's \& St Thomas' NHS Foundation Trust, London, UK

4 Department of Radiology, Guy's \& St Thomas' NHS Foundation Trust, London, UK

5 Department of Radiology, Imaging 2, Level 2, Lambeth Wing, St Thomas' Hospital, London SE1 7EH, UK
Main Messages

- Sarcopenia, sarcopenic obesity and visceral obesity are adverse prognostic factors in cancer patients.

- CT and MRI are the current gold standard in body composition evaluation.

- Body composition may affect chemotherapy tolerance and toxicities.

Keywords Sarcopenia - Sarcopenic obesity $\cdot$ CT $\cdot$ MRI · DXA

\section{Introduction}

Body composition is an important feature in cancer patients as it may affect the efficacy and toxicity of chemotherapy, and it is associated with patient outcomes in terms of functional status, surgical complication rates, length of hospital stay (LOS) and overall survival (OS) [1-8]. Assessment of body composition typically includes the quantitation of fat and muscle mass. In cancer patients, identification of risk factors including obesity (an increase in fat mass, in particular visceral fat mass), sarcopenia (loss of lean muscle mass and function) and sarcopenic obesity (a combination of loss of lean muscle mass and visceral obesity) will allow early supportive care such as dietary and/or physiotherapy interventions to be implemented.

\section{Obesity}

The World Health Organisation body mass index (BMI) is most commonly used to define obesity [9]. The BMI is calculated by weight (in kilograms, $\mathrm{kg}$ ) divided by the height (in metres, m) squared, where $\geq 40.0 \mathrm{~kg} / \mathrm{m}^{2}$ equates to morbid obesity, $35.0-39.9 \mathrm{~kg} / \mathrm{m}^{2}$ equates to class II obesity, 30.0 
$34.9 \mathrm{~kg} / \mathrm{m}^{2}$ equates to class I obesity and $25.0-29.9 \mathrm{~kg} / \mathrm{m}^{2}$ refers to overweight individuals [9]. However, associations between BMI and long-term outcomes and prognosis are weak in comparison to visceral obesity in cancer patients $[5$, $6,10,11]$. In the non-oncological setting, the waist circumference (WC) and waist-to-hip ratio (WHR) have been found to be better discriminators of diabetes and cardiovascular risks compared to BMI $[12,13]$. In the oncological setting, WC and WHR have been found to be associated with increased risk of endometrial, oesophagogastric, colorectal and breast cancers [14-16] although conflicting results were obtained in prostate and bladder cancers $[14,17]$. There is a suggestion that WC and WHR are associated with inferior oncological outcomes such as colorectal cancer [18].

Visceral obesity refers more specifically to the excessive accumulation of visceral fat in the abdominal cavity $[10,11$, 19, 20]. There is no definite normal range of visceral adipose tissue as this varies with age, gender, race and coexisting medical conditions [21]. Nonetheless, in one study that evaluated visceral adiposity on MRI in a predominantly Caucasian population, the 25th and 75th percentiles of intra-abdominal fat area were found to be $67.6-140.1 \mathrm{~cm}^{2}$ and $106.3-189.5 \mathrm{~cm}^{2}$ in females and males respectively [22]. Visceral obesity is calculated as the ratio of the visceral fat area to subcutaneous fat area, where a ratio greater than 0.4 is considered as visceral obesity [19]. Visceral fat differs from subcutaneous fat in that it has a higher number of large adipocytes, more glucocorticoid and androgen receptors, and is able to produce more free fatty acids in comparison to subcutaneous fat [19, 23, 24]. Visceral fat also secretes more bioactive molecules [13] and is associated with lower insulin sensitivity and higher circulating triglyceride levels [23-25] compared to subcutaneous fat. The link between visceral obesity and adverse outcomes in cancer patients may be partly due to increased insulin resistance and its influence on levels of endocrine hormonal secretion, which is also associated cancer progression [26, 27].

\section{Sarcopenia}

Sarcopenia literally translates as 'lack of' (penia) 'flesh' (sarco) in Greek but refers to a loss of muscle mass as well as function. Sarcopenia may be primary (age-related) or secondary (associated with reduced activity, poor nutrition, malabsorption, endocrine disease, neurodegenerative disorders or cancer cachexia) [28-31]. Most frequently, sarcopenia has been defined as an appendicular skeletal muscle mass less than two standard deviations below the mean of a young healthy adult group as determined by dual-energy X-ray absorptiometry (DXA) [28, 30]. The European Society of Parenteral and Enteral Nutrition Special Interest Group (ESPEN SIG) proposed that sarcopenia should be diagnosed based on the presence of two criteria: (1) low muscle mass and (2) impaired muscular function [32]. Due to the significant variation in body composition between males and females, sex-specific skeletal muscle index cut-offs $\left(52.4 \mathrm{~cm}^{2} / \mathrm{m}^{2}\right.$ and $38.5 \mathrm{~cm}^{2} / \mathrm{m}^{2}$ for males and females, respectively) to define sarcopenia have been proposed in cancer patients and were shown to be associated with mortality [1]. It is worth bearing in mind that these definitions were derived from computed tomography (CT) images obtained at the level of the L3 lumbar vertebra. The use of these sex-specific cut-offs has been supported by an international consensus on the definition of cancer cachexia in 2011 [33]. It is important to be aware that sarcopenia may be present even in the absence of weight loss. For example, Prado et al. showed that $15 \%$ of obese (defined as BMI $\geq 30 \mathrm{~kg} / \mathrm{m}^{2}$ ) cancer patients were sarcopenic [1].

\section{Sarcopenic obesity}

The combination of sarcopenia and obesity is classified as sarcopenic obesity. There are subtle variations in the exact definition of both conditions in various studies, depending on the method of assessment, although the latter is commonly defined as BMI $\geq 30 \mathrm{~kg} / \mathrm{m}^{2}$ [34]. Several factors could increase the risk of sarcopenic obesity [34]. Age-related body composition changes with progressive decline in muscle mass and/or strength is a significant risk factor [34, 35]. Hormonal changes, sedentary lifestyle and malnutrition may also occur in the elderly, contributing to sarcopenic obesity. In addition, adipose tissue secretes pro-inflammatory cytokines and adipokines, promoting insulin resistance [34, 35], and these pro-inflammatory markers can contribute towards low muscle mass and obesity [36].

\section{Cachexia}

Cachexia, derived from the Greek words 'cac' or bad and 'hexis' or condition, is well recognised in patients with chronic illnesses such as cancer, end-stage renal disease, cirrhosis and chronic obstructive pulmonary disease [30, 33]. The ESPEN SIG and Cachexia Consensus Working Group have defined cachexia as a complex metabolic syndrome in chronically ill patients, associated with loss of muscle mass with or without loss of fatty tissue [32, 37]. Many proposed factors are involved in the development of cachexia such as chronic inflammation, increased muscle protein breakdown and insulin resistance [33, 37]. Although it may be difficult to differentiate sarcopenia from cachexia particularly in the oncological setting, most cachectic individuals have sarcopenia but not all sarcopenic patients are cachectic [30,32].

\section{Assessment of body composition in clinical practice}

Various techniques may be used to estimate body composition. These include bioimpedance analysis (BIA), DXA, CT 
and magnetic resonance imaging (MRI). $\mathrm{CT}$ and MRI are currently considered the gold standards for estimating muscle mass [30]. Both imaging modalities are obtained as part of the standard patient care pathway from tumour staging to response assessment and surveillance, thus providing an excellent opportunity to integrate body composition assessment into current patient care. The merits and disadvantages of the various techniques are summarised in Table 1.

\section{Anthropometrics and bioimpedance analysis}

Anthropometric methods such as skin fold thickness by caliper measurement, mid arm and calf circumferences have been used to assess muscle mass. However, these methods are prone to measurement error with significant interobserver variability and are not recommended for routine diagnosis of sarcopenia [30].

BIA may be used to estimate fat mass relative to lean body mass [30]. This involves placing electrodes on the skin, e.g. of the hand and foot, and measuring the impedance of an applied low level electric current. The impedance is higher for fat and bone compared with soft tissue [30]. Impedance measurement can be affected by hydration status [38] and thus BIA should be performed under standard conditions to minimise the measurement variation.

A Japanese group has proposed sex-specific equations to estimate the appendicular skeletal mass using BIA: $0.197 \times$ (impedance index $)+0.179 \times($ weight $)-0.019$ (males) and $0.221 \times($ impedance index $)+0.117 \times($ weight $)+0.881$ (females) [39]. However, it should be noted that these equations were derived from the older Japanese population and have not been validated in other populations or in the oncological setting. The same group also defined the skeletal muscle mass index as appendicular skeletal mass/height ${ }^{2}[40]$. They classified those with a skeletal muscle mass index less than $7.09 \mathrm{~kg} / \mathrm{m}^{2}$ in males and $5.91 \mathrm{~kg} / \mathrm{m}^{2}$ in females as sarcopenic based on the lowest sex-specific $20 \%$ quintiles in the healthy population [40].

\section{Dual-energy X-ray absorptiometry}

Dual energy X-ray absorptiometry (DXA) exploits the difference in the attenuation of tissue and bone at different X-ray energies to measure lean body mass (LBM), fat mass (FM) and bone mineral mass (BMM) [41], which can be extrapolated to the whole body (Fig. 1). An X-ray source produces a fan beam at two average X-ray energies, typically $30-40 \mathrm{keV}$ and $70-90 \mathrm{keV}$, depending on whether filtration or $70 \mathrm{kV} /$ $140 \mathrm{kV}$ tube voltage switching is used [42]. The typical radiation exposure of a DXA scan is low $(0.1 \mathrm{mSv})$. The X-ray attenuation and transmission reflect the differences in tissue thickness, density and the elemental composition of the different compartments. Attenuation increases with tissue thickness and is greater for bone than soft tissue.

DXA is widely used as a clinical tool but is associated with some limitations. The DXA scan produces a two-dimensional image; therefore distinction between subcutaneous and visceral adipose tissue cannot be made. Certain assumptions have to be made, such as the extent of distribution of the fat and muscle compartments, particularly where there is overlying bone. As with any quantitative imaging technique, different manufacturers' software, calibration methods, calculation algorithms and scanners may result in variation in the calculated estimates of body composition [41, 43-46].

Table 1 Summary of the various techniques used in body composition analysis

\begin{tabular}{lll}
\hline Techniques & Advantages & Disadvantages \\
\hline Bioelectrical impedance analysis (BIA) & Inexpensive & Lack of precision \\
& Portable & Less time consuming \\
& No radiation exposure & Skeletal muscle quality cannot be analysed \\
& Immediate results & \\
Dual-energy X-ray absorptiometry (DXA) & Inexpensive & \\
& Low radiation exposure (equivalent to & Lack of portability \\
& Two-dimensional data \\
& More sensitive than BIA & Low precision compared to CT and MRI \\
& & Distinction between subcutaneous and \\
visceral adipose tissue cannot be made & Skeletal muscle quality cannot be analysed \\
Computed tomography (CT) & High accuracy and reproducible results & Radiation exposure \\
& Lean body mass, subcutaneous fat and & More expensive compared to BIA \& DXA \\
Magnetic resonance imaging (MRI) & visceral fat can be defined & Skeletal muscle quality cannot be assessed \\
& Best spatial resolution and body & More expensive compared to BIA and DXA \\
& mass composition differentiation & Longer image acquisition time \\
& No radiation exposure & Contraindications to MRI may preclude some patients \\
& & Skeletal muscle quality cannot be analysed
\end{tabular}




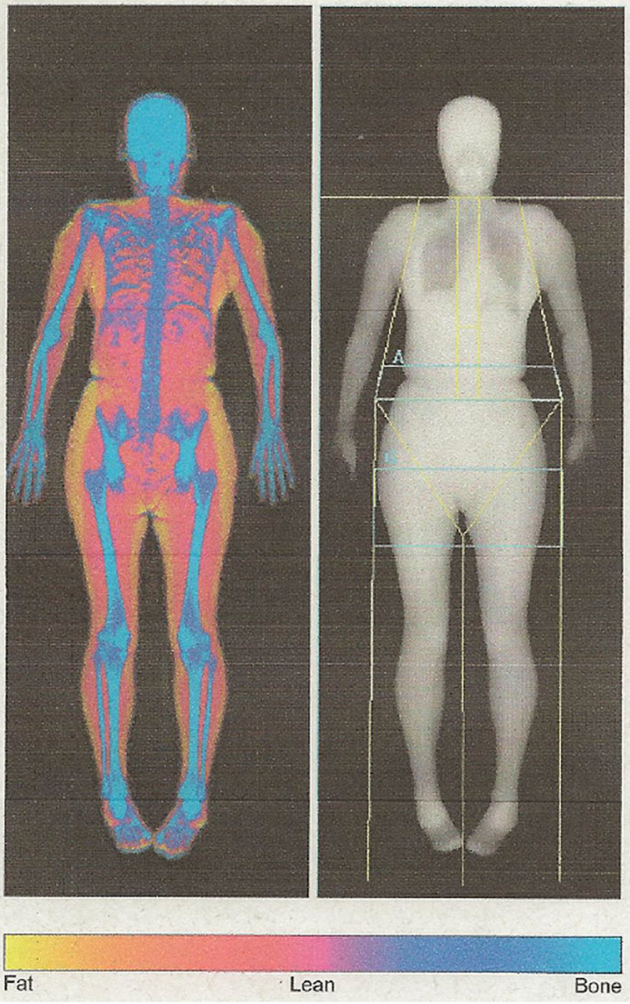

Fig. 1 Whole-body DXA image showing lean, fat and bone masses

\section{Computed tomography and magnetic resonance imaging}

CT and MRI are high spatial and contrast resolution crosssectional techniques that can provide estimates of lean muscle mass and adipose tissue as well as fat infiltration within the skeletal muscle [47-50]. The methods used in the measurement of cross-sectional body composition are similar for CT and MRI. The user is usually required to manually delineate the fat or muscle compartment of interest on a dedicated software platform (Fig. 2). These regions of interest are then further refined using specific Hounsfield unit (HU) segmentation thresholds in CT [1] or grey-level value thresholds in MRI, the latter requiring more complicated segmentation algorithms [51, 52].

\section{$C T$}

The performance of CT in body composition analysis has been shown to be superior to DXA [49]. Lean body mass, subcutaneous and visceral fat mass can be delineated for a given volume on $\mathrm{CT}$ images (Fig. 2) and extrapolated to the whole body [49]. Several parameters such as the total fat mass, total lean body mass, fat and lean body mass indices (normalised for stature), subcutaneous fat-to-muscle ratio and visceral-to-subcutaneous adipose tissue ratio may also be derived.

Total body fat mass and lean body mass may be defined using the following equations [49]:

Total body fat mass $(\mathrm{kg})=0.042 \times\left[\right.$ total adipose tissue at L3 $\left.\left(\mathrm{cm}^{2}\right)\right]+11.2$ Total body lean body mass $(\mathrm{kg})=0.3 \times\left[\right.$ skeletal muscle at L3 $\left.\left(\mathrm{cm}^{2}\right)\right]+6.06$

The L3 lumbar vertebra landmark is often used in crosssectional body composition analysis and is found to correspond to the whole-body tissue measurements [50, 53]. The field of view at this vertebral level includes the psoas, paraspinal muscles (erector spinae, quadratus lumborum) and abdominal wall muscles (transversus abdominus, external and internal obliques, rectus abdominus), thus making it an optimal level for skeletal muscle quantification. However, while the L3 vertebral level is also used to assess fat mass, the amount of fat will vary according to sex, age and body level. Thus, there are suggestions that visceral fat should be derived by obtaining measurements at several different anatomic levels [54] although others have found no significant clinical impact when correlating visceral fat measured at L2L3, L4-L5 and mid waist levels and patient outcome [55].

There is no defined guideline on the image acquisition parameters that are required for the purpose of crosssectional body composition analysis. Thus, for patients who are undergoing abdominopelvic $\mathrm{CT}$ as part their routine diagnostic or management algorithm, the following standard CT acquisition parameters are appropriate: $120 \mathrm{kV}$, variable mA with dose modulation, soft tissue reconstruction algorithm,
Fig. 2 Subcutaneous fat, visceral fat and skeletal muscle as depicted on an axial CT image at the level of L3 vertebral body
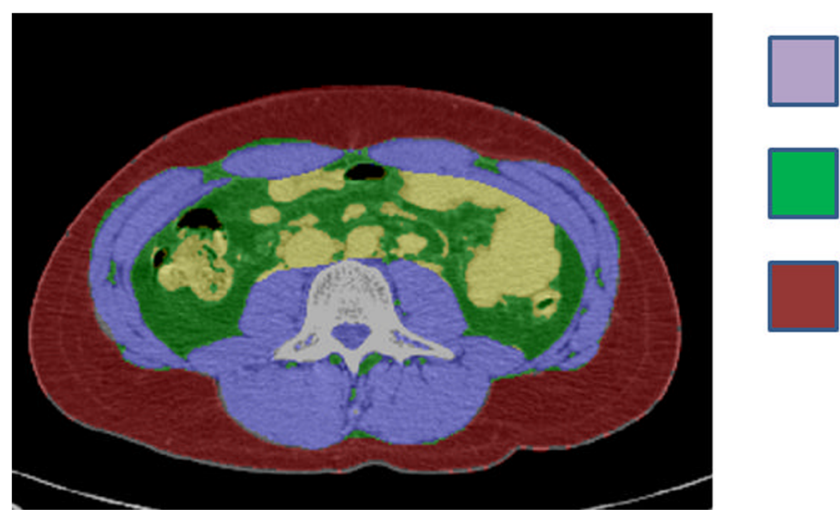

Skeletal muscle

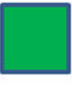

Visceral fat

Subcutaneous fat 
matrix of $512 \times 512$, field of view (FOV) of $30-35 \mathrm{~cm}$ and reconstructed slice thickness $5 \mathrm{~mm}$. However, for patients undergoing a targeted CT solely for the assessment of body composition, a limited low-dose axial 10-mm acquisition at the L3 level may be appropriate: $120 \mathrm{kV},<80 \mathrm{mAs}$, soft tissue reconstruction algorithm, matrix of $512 \times 512$ and FOV of 30$35 \mathrm{~cm}$ with the advantage that the additional radiation exposure from a limited CT is small and is equivalent to a chest radiograph [56].

\section{MRI}

The major advantage of MRI over CT in body composition analysis is its lack of radiation exposure. However, the use of MRI is limited by the local availability and technical expertise. Nonetheless, clinical MRI scanners are more widely available now and whole-body MRI techniques are being introduced that could represent a step forward in MRI assessment of body composition. MRI has better soft tissue definition particularly of adipose tissue compared to $\mathrm{CT}$ as fat has short $\mathrm{T} 1$ and long $\mathrm{T} 2$ proton relaxation times [57] and thus may improve image segmentation of adipose tissues and skeletal muscle. The majority of the published literature on the use of MRI has evaluated its use in fat mass analysis [52, 58, 59]. MRI estimation of subcutaneous and intra-abdominal adipose tissues has been shown to correlate with direct measurement of the corresponding cadaveric tissues [60]. Similar to CT, MRI evaluation at the level of the L2/3 vertebra was found to be a reliable estimate of fat mass [61].

Improved segmentation of MRI fat and lean body mass may be produced using a two-point DIXON method for fat/ water separation (Fig. 3) [62, 63]. The information from inand out-of-phase gradient echo sequences may be combined. In the in-phase image, the signal $\left(\mathrm{S}_{\mathrm{ip}}\right)$ represents the sum of fat $\left(\mathrm{S}_{\mathrm{f}}\right)$ and water $\left(\mathrm{S}_{\mathrm{w}}\right)$ signals, i.e. $\mathrm{S}_{\mathrm{ip}}=\mathrm{S}_{\mathrm{w}}+\mathrm{S}_{\mathrm{f}}$, while the out-ofphase signal represents the difference, i.e. $S_{i p}=S_{w}-S_{f}$. Averaging of the sum and difference of the in- and out-ofphase images will result in water and fat signal respectively. A correction for $\mathrm{T}^{*}$ differences is required as the two images have different echo times. The two-point DIXON method assumes that the main field $\mathrm{B}_{0}$ homogeneity is perfect.
However, this is not true and refinements such as a threepoint method have been proposed where an in-phase TE sequence is used to correct for $\mathrm{B}_{0}$ field homogeneity [64].

A simpler and quicker way of assessing whole-body fat mass and possibly lean body mass may be feasible with the advent of whole-body MRI and newer computer-aided methods of image segmentation [52]. This may have potential clinical utility as whole-body MRI is currently being evaluated as a staging modality in different tumour sites such as multiple myeloma.

A general limitation of these imaging techniques is that they only provide anatomical information and not functional information such as muscle function. Thus, these imaging findings have to be considered in conjunction with formal assessment of muscle function, particularly in the diagnosis of sarcopenia [32]. However, there is a suggestion that skeletal muscle attenuation on CT (Hounsfield units, HU) may potentially be a surrogate for muscle function $[65,66]$, with reduced $\mathrm{HU}$ within skeletal muscle representing increased intramuscular lipid deposition, which has been observed in those with neuromuscular disease [65]. This is still an area of research and no definite HU cut-offs have been reliably identified to represent reduced muscle function for this to be adopted in the clinical setting at present. Reduced skeletal muscle attenuation has also been found to be a negative prognostic factor in patients with gastrointestinal and respiratory tract cancers [67].

\section{Clinical applications in oncology}

\section{Body composition as a prognosis marker}

To our knowledge, there is no published study looking at the use of MRI body composition assessment in cancer patients. $\mathrm{CT}$ is the most commonly used cross-sectional imaging technique in this setting and we will be focusing on its use in this section.

Sarcopenic cancer patients have been shown to have higher rates of morbidity and mortality $[1,4,6,8,10,48]$. Lieffers et al. showed that patients with colorectal cancer were at risk
Fig. 3 T1-weighted axial DIXON MRI images highlighting (a) fat and (b) water signals at the level of the L3 vertebral body

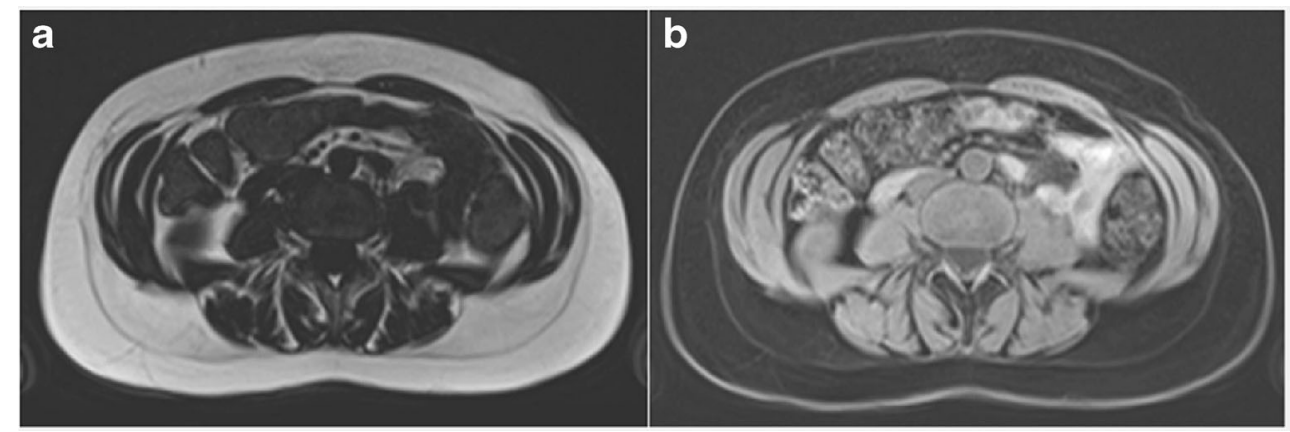


of adverse outcomes after primary colorectal surgery if they had co-existing sarcopenia [4]. In this study, more than a third of the patients (39\%) were found to be sarcopenic and they had an increased length of stay in hospital (mean 16 \pm 14 days) compared to the non-sarcopenic patients $(12 \pm 10$ days, $p=$ $0.038)$. Post-operative infection risk was also higher in those with sarcopenia ( $24 \%$ vs. $13 \%, p=0.025$ ). These risks were more pronounced in patients aged 65 years and above. However, visceral and subcutaneous adiposities were not significant predictors of length of stay and postoperative complications in a separate study [5].

Moon et al. showed that the visceral fat area-tosubcutaneous fat area ratio was a significant prognostic factor in predicting disease-free survival in patients with resectable colorectal cancer [6]. Those with visceral fat area-tosubcutaneous fat area ratio $>0.5$ had shorter disease-free survival (HR 1.98, $95 \%$ CI 1.02-3.87, $p=0.044$ ) although it did not have a significant impact on overall survival. Similarly, viscerally obese patients (visceral fat area-to-subcutaneous fat area ratio $\geq 0.4$ ) with rectal cancer had poorer disease-free survival (HR 3.50, $95 \%$ CI $1.12-10.17, p=0.09$ ) but there was no significant difference in overall survival [10]. In contrast, body mass index measurements did not correlate with any survival outcomes $[6,10]$.

Prado et al. found that sarcopenic obesity was a significant prognostic factor in patients with gastrointestinal and respiratory tract cancers [1]. Patients with coexisting sarcopenic obesity had poorer functional status $(p=0.009)$ and overall survival (HR 4.2, $95 \%$ CI 2.4-7.2, $p<0.0001$ ). These findings were confirmed in patients with advanced pancreatic cancer [48]. In this study, sarcopenic obesity was a significant predictor of reduced overall survival (HR 2.07, $95 \% \mathrm{CI}=1.23-$ $3.50, p=0.006$ ).

In contrast to the above positive findings, fat mass and fatfree mass were not associated with in-hospital mortality or survival in patients with gastro-oesophageal (GOJ) cancer treated with neoadjuvant chemotherapy [68]. However, it is noteworthy that the authors evaluated the prognostic value of pre-treatment and post-treatment fat-free mass only. Whether fat mass, as shown in the colorectal cancer population, could have a greater prognostic impact in GOJ cancer remains unclear. In addition, it may be that a reduction in fat-free mass during treatment may be a more important prognostic factor than the absolute baseline or post-treatment values as evaluated in this study.

\section{Treatment implications}

In addition to its potential prognostic impact, body composition may also affect an individual's tolerance to non-surgical treatment and could be predictive of treatment toxicity. First, the use of chemotherapy has been shown to alter body composition [69]. Yip et al. showed that fat mass and fat-free mass, as measured using CT, and weight decreased after neoadjuvant chemotherapy in patients with oesophageal cancer (Fig. 4). Similarly, a separate study demonstrated that fat mass and fat-free mass decreased but the proportion of patients with sarcopenic obesity increased following neoadjuvant chemotherapy in those with GOJ cancers [68].

These body composition changes could have an important impact on patient's tolerance to subsequent therapy as sarcopenia may increase the risk of chemotherapy toxicity $[2,3,70]$. At present, the body surface area is used to calculate cytotoxic chemotherapy dosing. As with the body mass index, the body surface area is derived using the patient's height and weight but is associated with many limitations, particularly in those with extreme variation in body composition such as obese patients, leading to under- or overdosing [71]. The risk of toxicity is higher in female patients as they tend to have a lower lean body mass in comparison to their body surface area [2]. This has led to recent suggestions that lean body mass may be a better measure to dose chemotherapy on an individual patient basis $[2,70]$.

Prado et al. showed that lean body mass was a significant predictor of dose-limiting toxicities in patients treated with 5fluorouracil (5FU) and leucovorin for stage II/III colon cancer [2]. In this study, females who had more than $20 \mathrm{mg}$ of 5FU/ $\mathrm{kg}$ lean body mass were found to have lower cross-sectional muscle mass and LBM on CT. The authors found that a 5FU/ $\mathrm{kg}$ lean body mass cut-off value of less than $20 \mathrm{mg} / \mathrm{kg}$ was a

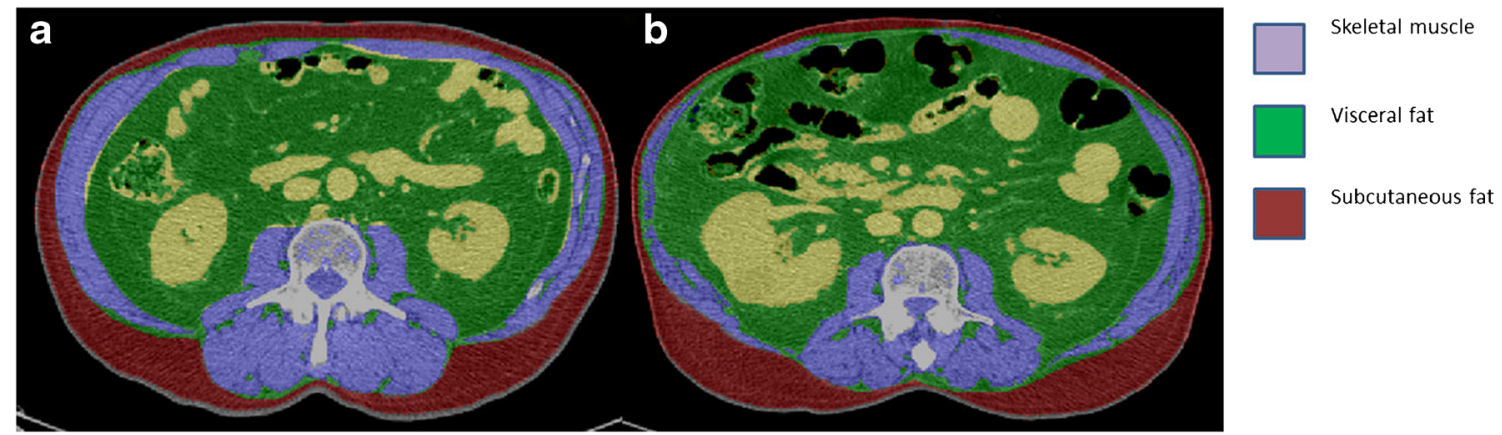

Fig. 4 Axial CT images at the level of L3 vertebra demonstrating progressive sarcopenia in a patient with oesophageal cancer before (a) and after (b) neoadjuvant chemotherapy. There is a loss of abdominal muscle mass with an increase in visceral fat 
significant predictor of toxicity. This is possibly due to the differential drug distribution in the different body compartments as hydrophilic drugs are distributed into the lean body compartment whereas lipophilic drugs are distributed into the fat compartment. Thus, the size of these compartments, which could be easily assessed using CT, would affect drug distribution and therefore toxicity.

The same group also evaluated the impact of sarcopenia on toxicity and time to progression in patients with metastatic breast cancer treated with capecitabine [70]. They found that $50 \%$ of sarcopenic patients had grade 2 or greater toxicities compared to $20 \%$ of non-sarcopenic patients $(p=0.03)$. Sarcopenic patients also had significantly shorter time to progression compared to the non-sarcopenic cohort (median 62 days vs. 105 days, $p=0.05$ ). Similar observations were also noted in patients treated with sorafenib for metastatic renal cell carcinoma [3]. In this study, a greater proportion of sarcopenic male patients experienced dose-limiting toxicities during sorafenib therapy compared to the non-sarcopenic patients ( $37 \%$ vs. $5 \%, p<0.04)$.

An objective assessment of body composition using crosssectional imaging techniques such as CT and MRI has the potential to complement our current clinical and nutritional evaluation of patients' fitness and treatment tolerability. This information can be readily obtained from standard diagnostic scans performed during the various stages of patient care. Nutritional support can then be initiated at an earlier and appropriate stage, which could improve treatment compliance and clinical outcome.

\section{Other metabolic associations}

Although not a direct oncological implication, the metabolic effect of body composition on cardiovascular risk and mortality will have an impact on the patients' overall life expectancy and tolerance to oncological treatment. Anthropometric indices such as BMI, WC and WHR are associated with cardiovascular risk factors such as hypertension, diabetes and dyslipidemia, and cardiovascular disease [12, 72]. Similarly, visceral adipose tissue as defined on CT has been shown to be associated with adverse cardiovascular risk factors [25]. These associations should be considered in order to provide a holistic approach to patient care.

\section{Future directions}

There is sufficient evidence to support the use of body composition assessment to direct and improve supportive oncological care such as dietary intervention and physiotherapy support. As cross-sectional body composition evaluation is straight forward, this can be introduced with relative ease into a routine oncology report particularly in high-risk patients with gastrointestinal cancers or pre-existing gastrointestinal disease. However, the use of body composition in modifying cancer therapy requires further research preferably as prospective clinical studies.

\section{Conclusions}

In conclusion, sarcopenia, sarcopenic obesity and visceral obesity may be associated with negative oncological outcomes. Imaging assessment of body composition can be readily applied in the clinical setting with the potential to improve individual nutritional care and perhaps chemotherapy dose calculation. This personalised cancer management strategy may reduce treatment-related toxicities and ultimately improve patient outcomes.

Funding disclosure This work was supported by financial support from the Department of Health via the National Institute of Health Research Biomedical Research Centre award to Guy's and St Thomas' NHS Foundation Trust in partnership with King's College London and King's College Hospital NHS Foundation Trust and from the Comprehensive Cancer Imaging Centre, funded by the Cancer Research UK and Engineering and Physical Sciences Research Council in association with the Medical Research Council and Department of Health (England).

Connie Yip receives funding support from the National Medical Research Council, Singapore.

Open Access This article is distributed under the terms of the Creative Commons Attribution 4.0 International License (http:// creativecommons.org/licenses/by/4.0/), which permits unrestricted use, distribution, and reproduction in any medium, provided you give appropriate credit to the original author(s) and the source, provide a link to the Creative Commons license, and indicate if changes were made.

\section{References}

1. Prado CM, Lieffers JR, McCargar LJ et al (2008) Prevalence and clinical implications of sarcopenic obesity in patients with solid tumours of the respiratory and gastrointestinal tracts: a population-based study. Lancet Oncol 9(7):629-635

2. Prado CM, Baracos VE, McCargar LJ et al (2007) Body composition as an independent determinant of 5-fluorouracil-based chemotherapy toxicity. Clin Cancer Res 13(11):3264-3268

3. Antoun S, Baracos VE, Birdsell L, Escudier B, Sawyer MB (2010) Low body mass index and sarcopenia associated with dose-limiting toxicity of sorafenib in patients with renal cell carcinoma. Ann Oncol 21(8):1594-1598

4. Lieffers JR, Bathe OF, Fassbender K, Winget M, Baracos VE (2012) Sarcopenia is associated with postoperative infection and delayed recovery from colorectal cancer resection surgery. Br J Cancer 107(6):931-936

5. Cecchini S, Cavazzini E, Marchesi F, Sarli L, Roncoroni L (2011) Computed tomography volumetric fat parameters versus body mass index for predicting short-term outcomes of colon surgery. World J Surg 35(2):415-423 
6. Moon HG, Ju YT, Jeong CY et al (2008) Visceral obesity may affect oncologic outcome in patients with colorectal cancer. Ann Surg Oncol 15(7):1918-1922

7. Thibault R, Genton L, Pichard C (2012) Body composition: why, when and for who? Clin Nutr 31(4):435-447

8. Balentine CJ, Enriquez J, Fisher W et al (2010) Intra-abdominal fat predicts survival in pancreatic cancer. J Gastrointest Surg 14(11): $1832-1837$

9. World Health Organization (2000) Obesity: preventing and managing the global epidemic. Report of a WHO consultation. World Health Organ Tech Rep Ser, 894:i-xii, 1-253

10. Clark W, Siegel EM, Chen YA et al (2013) Quantitative measures of visceral adiposity and body mass index in predicting rectal cancer outcomes after neoadjuvant chemoradiation. J Am Coll Surg 216(6):1070-1081

11. Rickles AS, Iannuzzi JC, Mironov O et al (2013) Visceral obesity and colorectal cancer: are we missing the boat with BMI? J Gastrointest Surg 17(1):133-143, discussion p 143

12. Lee CM, Huxley RR, Wildman RP, Woodward M (2008) Indices of abdominal obesity are better discriminators of cardiovascular risk factors than BMI: a meta-analysis. J Clin Epidemiol 61(7):646-653

13. Zimmet P, Magliano D, Matsuzawa Y, Alberti G, Shaw J (2005) The metabolic syndrome: a global public health problem and a new definition. J Atheroscler Thromb 12(6):295-300

14. Harding JL, Shaw JE, Anstey KJ et al (2015) Comparison of anthropometric measures as predictors of cancer incidence: A pooled collaborative analysis of 11 Australian cohorts. Int J Cancer. doi:10. 1002/ijc. 29529

15. Aune D, Navarro Rosenblatt DA, Chan DS et al (2015) Anthropometric factors and endometrial cancer risk: a systematic review and dose-response meta-analysis of prospective studies. Ann Oncol. doi:10.1093/annonc/mdv142

16. Steffen A, Huerta JM, Weiderpass E et al (2015) General and abdominal obesity and risk of esophageal and gastric adenocarcinoma in the European Prospective Investigation into Cancer and Nutrition. Int J Cancer 137(3):646-657

17. Roswall N, Freisling H, Bueno-de-Mesquita HB et al (2014) Anthropometric measures and bladder cancer risk: a prospective study in the EPIC cohort. Int J Cancer 135(12):2918-2929

18. Fedirko V, Romieu I, Aleksandrova K et al (2014) Pre-diagnostic anthropometry and survival after colorectal cancer diagnosis in Western European populations. Int J Cancer 135(8):1949-1960

19. Matsuzawa Y, Nakamura T, Shimomura I, Kotani K (1995) Visceral fat accumulation and cardiovascular disease. Obes Res 3(Suppl 5): $645 \mathrm{~S}-647 \mathrm{~S}$

20. Tsujinaka S, Konishi F, Kawamura YJ et al (2008) Visceral obesity predicts surgical outcomes after laparoscopic colectomy for sigmoid colon cancer. Dis Colon Rectum 51(12):1757-1765, discussion 1765-1757

21. Hans TS, Seidell JC, Currall JE, Morrison CE, Deurenberg P, Lean ME (1997) The influences of height and age on waist circumference as an index of adiposity in adults. Int J Obes Relat Metab Disord 21(1):83-89

22. Schreiner PJ, Terry JG, Evans GW, Hinson WH, Crouse JR 3rd, Heiss G (1996) Sex-specific associations of magnetic resonance imaging-derived intra-abdominal and subcutaneous fat areas with conventional anthropometric indices. The Atherosclerosis Risk in Communities Study. Am J Epidemiol 144(4):335-345

23. Matsuzawa Y, Shimomura I, Nakamura T, Keno Y, Kotani K, Tokunaga K (1995) Pathophysiology and pathogenesis of visceral fat obesity. Obes Res 3(Suppl 2):187S-194S

24. Ibrahim MM (2010) Subcutaneous and visceral adipose tissue: structural and functional differences. Obes Rev 11(1):11-18

25. Fox CS, Massaro JM, Hoffmann U et al (2007) Abdominal visceral and subcutaneous adipose tissue compartments: association with metabolic risk factors in the Framingham Heart Study. Circulation 116(1):39-48

26. Otake S, Takeda H, Suzuki Y et al (2005) Association of visceral fat accumulation and plasma adiponectin with colorectal adenoma: evidence for participation of insulin resistance. Clin Cancer Res 11(10):3642-3646

27. van Kruijsdijk RC, van der Wall E, Visseren FL (2009) Obesity and cancer: the role of dysfunctional adipose tissue. Cancer Epidemiol Biomarkers Prev 18(10):2569-2578

28. Baumgartner RN, Koehler KM, Gallagher D et al (1998) Epidemiology of sarcopenia among the elderly in New Mexico. Am J Epidemiol 147(8):755-763

29. Goodpaster BH, Park SW, Harris TB et al (2006) The loss of skeletal muscle strength, mass, and quality in older adults: the health, aging and body composition study. J Gerontol A Biol Sci Med Sci 61(10):1059-1064

30. Cruz-Jentoft AJ, Baeyens JP, Bauer JM et al (2010) Sarcopenia: European consensus on definition and diagnosis: Report of the European Working Group on Sarcopenia in Older People. Age Ageing 39(4):412-423

31. Coker RH, Wolfe RR (2012) Bedrest and sarcopenia. Curr Opin Clin Nutr Metab Care 15(1):7-11

32. Muscaritoli M, Anker SD, Argiles J et al (2010) Consensus definition of sarcopenia, cachexia and pre-cachexia: joint document elaborated by Special Interest Groups (SIG) "cachexia-anorexia in chronic wasting diseases" and "nutrition in geriatrics". Clin Nutr 29(2):154-159

33. Fearon K, Strasser F, Anker SD et al (2011) Definition and classification of cancer cachexia: an international consensus. Lancet Oncol 12(5):489-495

34. Stenholm S, Harris TB, Rantanen T, Visser M, Kritchevsky SB, Ferrucci L (2008) Sarcopenic obesity: definition, cause and consequences. Curr Opin Clin Nutr Metab Care 11(6):693-700

35. Roubenoff R (2004) Sarcopenic obesity: the confluence of two epidemics. Obes Res 12(6):887-888

36. Schrager MA, Metter EJ, Simonsick E et al (2007) Sarcopenic obesity and inflammation in the InCHIANTI study. J Appl Physiol (1985) 102(3):919-925

37. Evans WJ, Morley JE, Argiles J et al (2008) Cachexia: a new definition. Clin Nutr 27(6):793-799

38. Thompson DL, Thompson WR, Prestridge TJ et al (1991) Effects of hydration and dehydration on body composition analysis: a comparative study of bioelectric impedance analysis and hydrodensitometry. J Sports Med Phys Fitness 31(4):565-570

39. Yoshida D, Shimada H, Park H et al (2014) Development of an equation for estimating appendicular skeletal muscle mass in Japanese older adults using bioelectrical impedance analysis. Geriatr Gerontol Int 14(4):851-857

40. Yoshida D, Suzuki T, Shimada H et al (2014) Using two different algorithms to determine the prevalence of sarcopenia. Geriatr Gerontol Int 14(Suppl 1):46-51

41. Albanese CV, Diessel E, Genant HK (2003) Clinical applications of body composition measurements using DXA. J Clin Densitom 6(2):75-85

42. Rothney MP, Brychta RJ, Schaefer EV, Chen KY, Skarulis MC (2009) Body composition measured by dual-energy X-ray absorptiometry half-body scans in obese adults. Obesity (Silver Spring) 17(6):1281-1286

43. Laskey MA (1996) Dual-energy X-ray absorptiometry and body composition. Nutrition 12(1):45-51

44. Duren DL, Sherwood RJ, Czerwinski SA et al (2008) Body composition methods: comparisons and interpretation. J Diabetes Sci Technol 2(6):1139-1146

45. Shepherd JA, Fan B, Lu Y et al (2012) A multinational study to develop universal standardization of whole-body bone density and 
composition using GE Healthcare Lunar and Hologic DXA systems. J Bone Miner Res 27(10):2208-2216

46. Tylavsky FA, Lohman TG, Dockrell M et al (2003) Comparison of the effectiveness of 2 dual-energy X-ray absorptiometers with that of total body water and computed tomography in assessing changes in body composition during weight change. Am J Clin Nutr 77(2): 356-363

47. Baracos VE, Reiman T, Mourtzakis M, Gioulbasanis I, Antoun S (2010) Body composition in patients with non-small cell lung cancer: a contemporary view of cancer cachexia with the use of computed tomography image analysis. Am J Clin Nutr 91(4):1133S$1137 \mathrm{~S}$

48. Tan BH, Birdsell LA, Martin L, Baracos VE, Fearon KC (2009) Sarcopenia in an overweight or obese patient is an adverse prognostic factor in pancreatic cancer. Clin Cancer Res 15(22):69736979

49. Mourtzakis M, Prado CM, Lieffers JR, Reiman T, McCargar LJ, Baracos VE (2008) A practical and precise approach to quantification of body composition in cancer patients using computed tomography images acquired during routine care. Appl Physiol Nutr Metab 33(5):997-1006

50. Mitsiopoulos N, Baumgartner RN, Heymsfield SB, Lyons W, Gallagher D, Ross R (1998) Cadaver validation of skeletal muscle measurement by magnetic resonance imaging and computerized tomography. J Appl Physiol (1985) 85(1):115-122

51. Seidell JC, Bakker CJ, van der Kooy K (1990) Imaging techniques for measuring adipose-tissue distribution-a comparison between computed tomography and 1.5-T magnetic resonance. Am J Clin Nutr 51(6):953-957

52. Brennan DD, Whelan PF, Robinson K et al (2005) Rapid automated measurement of body fat distribution from whole-body MRI. AJR Am J Roentgenol 185(2):418-423

53. Shen W, Punyanitya M, Wang Z et al (2004) Total body skeletal muscle and adipose tissue volumes: estimation from a single abdominal cross-sectional image. J Appl Physiol (1985) 97(6):2333-2338

54. Warren M, Schreiner PJ, Terry JG (2006) The relation between visceral fat measurement and torso level - is one level better than another? The Atherosclerosis Risk in Communities Study, 1990 1992. Am J Epidemiol 163(4):352-358

55. Balentine CJ, Marshall C, Robinson C et al (2010) Validating quantitative obesity measurements in colorectal cancer patients. J Surg Res 164(1):18-22

56. Thibault R, Pichard C (2012) The evaluation of body composition: a useful tool for clinical practice. Ann Nutr Metab 60(1):6-16

57. Foster MA, Hutchison JM, Mallard JR, Fuller M (1984) Nuclear magnetic resonance pulse sequence and discrimination of high- and low-fat tissues. Magn Reson Imaging 2(3):187-192

58. Fowler PA, Fuller MF, Glasbey CA et al (1991) Total and subcutaneous adipose tissue in women: the measurement of distribution and accurate prediction of quantity by using magnetic resonance imaging. Am J Clin Nutr 54(1):18-25
59. Machann J, Thamer C, Schnoedt B et al (2005) Standardized assessment of whole body adipose tissue topography by MRI. J Magn Reson Imaging 21(4):455-462

60. Abate N, Burns D, Peshock RM, Garg A, Grundy SM (1994) Estimation of adipose tissue mass by magnetic resonance imaging: validation against dissection in human cadavers. J Lipid Res 35(8): 1490-1496

61. Abate N, Garg A, Coleman R, Grundy SM, Peshock RM (1997) Prediction of total subcutaneous abdominal, intraperitoneal, and retroperitoneal adipose tissue masses in men by a single axial magnetic resonance imaging slice. Am J Clin Nutr 65(2):403-408

62. Coombs BD, Szumowski J, Coshow W (1997) Two-point Dixon technique for water-fat signal decomposition with B0 inhomogeneity correction. Magn Reson Med 38(6):884-889

63. Dixon WT (1984) Simple proton spectroscopic imaging. Radiology 153(1):189-194

64. Glover GH, Schneider E (1991) Three-point Dixon technique for true water/fat decomposition with B0 inhomogeneity correction. Magn Reson Med 18(2):371-383

65. Liu M, Chino N, Ishihara T (1993) Muscle damage progression in Duchenne muscular dystrophy evaluated by a new quantitative computed tomography method. Arch Phys Med Rehabil 74(5): 507-514

66. Visser M, Kritchevsky SB, Goodpaster BH et al (2002) Leg muscle mass and composition in relation to lower extremity performance in men and women aged 70 to 79 : the health, aging and body composition study. J Am Geriatr Soc 50(5):897-904

67. Martin L, Birdsell L, Macdonald N et al (2013) Cancer cachexia in the age of obesity: skeletal muscle depletion is a powerful prognostic factor, independent of body mass index. J Clin Oncol 31(12): $1539-1547$

68. Awad S, Tan BH, Cui H et al (2012) Marked changes in body composition following neoadjuvant chemotherapy for oesophagogastric cancer. Clin Nutr 31(1):74-77

69. Yip C, Goh V, Davies A et al (2014) Assessment of sarcopenia and changes in body composition after neoadjuvant chemotherapy and associations with clinical outcomes in oesophageal cancer. Eur Radiol 24(5):998-1005

70. Prado CM, Baracos VE, McCargar LJ et al (2009) Sarcopenia as a determinant of chemotherapy toxicity and time to tumor progression in metastatic breast cancer patients receiving capecitabine treatment. Clin Cancer Res 15(8):2920-2926

71. Griggs JJ, Mangu PB, Anderson H et al (2012) Appropriate chemotherapy dosing for obese adult patients with cancer: American Society of Clinical Oncology clinical practice guideline. J Clin Oncol 30(13):1553-1561

72. Huxley R, Mendis S, Zheleznyakov E, Reddy S, Chan J (2010) Body mass index, waist circumference and waist:hip ratio as predictors of cardiovascular risk-a review of the literature. Eur J Clin Nutr 64(1):16-22 